\title{
Amygdala-Prefrontal Disconnection in Borderline Personality Disorder
}

\author{
Antonia S New*, 1,2, Erin A Hazlett ${ }^{1,3}$, Monte S Buchsbaum ${ }^{1,3}$, Marianne Goodman ${ }^{1,2}$, Serge A Mitelman ${ }^{1,3}$, \\ Randall Newmark ${ }^{3}$, Roanna Trisdorfer', M Mehmet Haznedar',3, Harold W Koenigsberg ',2, Janine Flory' \\ and Larry J Siever ${ }^{1,2}$ \\ 'Department of Psychiatry, Mount Sinai School of Medicine, New York, NY, USA; ${ }^{2}$ Department of Psychiatry, Mount Sinai School of Medicine, \\ Bronx VA Medical Center, Bronx, NY, USA; ${ }^{3}$ Department of Psychiatry and Neuroscience PET Laboratory, Mount Sinai School of Medicine, \\ New York, NY, USA
}

Abnormal fronto-amygdala circuitry has been implicated in impulsive aggression, a core symptom of borderline personality disorder (BPD). We examined relative glucose metabolic rate (rGMR) at rest and after m-CPP (meta-chloropiperazine) with ${ }^{18}$ fluorodeoxyglucose (FDG) with positron emission tomography (PET) in 26 impulsive aggressive (IED)-BPD patients and 24 controls. Brain edges/amygdala were visually traced on MRI scans co-registered to PET scans; rGMR was obtained for ventral and dorsal regions of the amygdala and Brodmann areas within the prefrontal cortex (PFC). Correlation coefficients were calculated between rGMR for dorsal/ventral amygdala regions and PFC. Additionally, amygdala volumes and rGMR were examined in BPD and controls. Correlations PFClamygdala Placebo: Controls showed significant positive correlations between right orbitofrontal (OFC) and ventral, but not dorsal, amygdala. Patients showed only weak correlations between amygdala and the anterior PFC, with no distinction between dorsal and ventral amygdala. Correlations PFClamygdala: m-CPP response: Controls showed positive correlations between OFC and amygdala regions, whereas patients showed positive correlations between dorsolateral PFC and amygdala. Group differences between interregional correlational matrices were highly significant. Amygdala volume/metabolism: No group differences were found for amygdala volume, or metabolism in the placebo condition or in response to meta-chloropiperazine ( $\mathrm{m}$-CPP). We demonstrated a tight coupling of metabolic activity between right OFC and ventral amygdala in healthy subjects with dorsoventral differences in amygdala circuitry, not present in IED-BPD. We demonstrated no significant differences in amygdala volumes or metabolism between BPD patients and controls. Neuropsychopharmacology (2007) 32, 1629-1640; doi:I0.1038/sj.npp. I 30 I283; published online 3 January 2007

Keywords: amygdala; positron emission tomography; impulsive aggression

\section{INTRODUCTION}

\section{The Prefrontal-Amygdala Circuit}

The concept that the prefrontal cortex (PFC) controls and inhibits the amygdala and other limbic structures, termed 'the reptilian brain', was proposed many years ago (McLean, 1955). Abundant preclinical data indicate that areas of the PFC exert inhibitory control over the amygdala. A series of experiments in rats have shown that the medial PFC inhibits activity in the basolateral amygdala by stimulating inhibitory interneurons in the amygdala (Rosenkranz and Grace, 1999, 2002). Many other such studies that have shown this phenomenon in rodents (al Maskati and Zbrozyna, 1989;

*Correspondence: Dr AS New, Department of Psychiatry, Mount Sinai School of Medicine, Box 1218, One Gustave Levy Place, New York, NY 10029, USA, Tel: + I 2122410193 , Fax: + I 2128242302

E-mail: antonia.new@mssm.edu

Received 7 March 2006; revised 26 September 2006; accepted 2 October 2006
Halasz et al, 2002; Jinks and McGregor, 1997; McDonald and Mascagni, 1996; Morgan and LeDoux, 1995; Zbrozyna and Westwood, 1991). In primates, damage to the lateral PFC causes a loss of inhibitory control in attention tasks (Dias et al, 1996; Stefanacci and Amaral, 2002), whereas damage to orbital frontal cortex (OFC) causes a loss of inhibitory control in 'affective' processing and increased aggression (Izquierdo et al, 2005). In the macaque monkey, whereas both lateral and medial areas within OFC have strong association with limbic regions, lateral OFC has specific connections to the amygdala (Carmichael and Price, 1995).

In human beings, functional brain imaging provides an approach in assessing the relationship between prefrontal and amygdala function by examining the correlation coefficients between activity in the two structures. Human studies with ${ }^{18}$ fluorodeoxyglucose (FDG)-positron emission tomography (PET) and fMRI have suggested a significant correlation between these two structures, but have primarily been carried out in healthy subjects. The directionality of 
these correlations has been inconsistent, with some studies showing positive correlations between areas of PFC and amygdala (Pezawas et al, 2005), and other studies showing negative correlations (Hariri et al, 2000, 2003). Specifically, one fMRI study during a perceptual task involving viewing pictures of frightening faces showed significant negative correlation between rostral anterior cingulate, but negative correlations between amygdala and caudal anterior cingulate (Pezawas et al, 2005), whereas another study with a similar task showed a negative correlation between right PFC and amygdala activity (Hariri et al, 2000). Significant negative correlations were also identified between the response of the left amygdala and those of the right OFC as well as the anterior cingulate (Hariri et al, 2003). An fMRI study of surprised faces show increased activation of the right ventral amygdala when the subject interpreted the face negatively, whereas a positive interpretation of the face yielded activation of OFC (Kim et al, 2003). A subsequent study by the same group showed activation of lateral OFC in response to negative $v s$ positive sentences, whereas medial OFC was activated in response to positive $v s$ negative sentences (Kim et al, 2004), suggesting that subregions of OFC may play different roles in relation to amygdala function.

Studies of patients with affective disorder (Drevets et al, 1992) and post-traumatic stress disorder (Shin et al, 2005) have tended to find negative correlations between specifically medial PFC and amygdala in patients but not controls, suggesting coupling only in psychopathology. Recent data suggest a role for serotonin in modulating connectivity between PFC and the amygdala, with subjects carrying the 'short' allele of the serotonin transporter gene (a gene possibly conferring a risk for mood disorders), showing greater fMRI amygdala/medial OFC 'coupling' during an emotional picture viewing task than those with only the 'long' allele (Hariri et al, 2006; Heinz et al, 2005; Pezawas et al, 2005).

\section{Subregions of the Amygdala in Emotion}

Subregions of the human amygdala have been shown to have specific functions, roughly organized on a dorsoventral dimension. The ventral amygdala includes primarily the basolateral complex (BLC) and the dorsal nucleus of the Central Nucleus (CN). Whalen et al (2001) suggest that the human dorsal $v s$ ventral designation within the amygdala provides a means for incorporating numerous results from the animal literature offering compelling evidence that the BLC (located ventrally in the human) can be dissociated behaviorally from the central nucleus (located dorsally in the human) and is the component of the amygdala predominantly involved in emotion modulation. Further, they note that whereas expressions of fear appear to activate the BLC and $\mathrm{CN}$ (ie ventral and dorsal amygdala), anger may involve the $\mathrm{CN}$ to a lesser degree (than the BLC) because less additional information concerning the stimulus is required (Whalen et al, 2001). Furthermore, fMRI studies by this group have shown that specifically the ventral amygdala is in response to emotional stimuli (Kim et al, 2003,2004 ). The ventral amygdala (basolateral amygdala in rats)-OFC circuit role in associative encoding and aversive odor was also supported in rat studies of amygdala activity in OFC (Saddoris et al, 2005; Schoenbaum et al, 2003).

The amygdala has been implicated not only in the processing of negative emotion in general, but also more specifically in the production of aggressive behavior in animal studies. Electric stimulation of the lateral nucleus of the amygdala in cats results in predatory attack behavior (Gregg and Siegel, 2001). In prairie voles, the medial nucleus of the amygdala has been shown to be involved in the regulation of aggression towards intruders (Wang et al, 1997). In primates, ablation of the amygdala bilaterally leads to increased social affiliation and decreased aggression (Emery et al, 2001; Meunier et al, 1999). These data have been taken to demonstrate a central role for the amygdala in the production of aggression, and led to the successful use of unilateral amygdalectomy in the treatment of a small number of cases of pathological aggression in human beings (Sachdev et al, 1992).

\section{Borderline Personality Disorder as a Prototype of Emotion Dysregulation}

Borderline personality disorder (BPD) is an illness, characterized by the symptom of emotional dysregulation and disinhibited anger, which often leads to aggressive behavior. The model of altered prefrontal-amygdala connectivity provides a model for the primary symptom in $\mathrm{BPD}$, disinhibition of emotion. To date, however, ours is the first study in BPD examining the relationship between prefrontal regions and amygdala in BPD.

A number of studies have reported abnormal PFC in BPD, as well as in impulsive aggressive subjects with a variety of personality disorders. An ${ }^{18}$ FDG-PET study reported reduced glucose metabolism in BPD patients compared to healthy controls in PFC, and anterior cingulate bilaterally (De La Fuente et al, 1997). In response to serotonergic challenge, specifically impulsive-aggressive BPD patients demonstrate decreased metabolism in anterior cingulate and PFC, compared to controls (New et al, 2002; Siever et al, 1999b; Soloff et al, 2003). Numerous studies have demonstrated decreased serotonergic responsiveness in impulsive aggressive patients with personality disorders (Coccaro, 1989; Dougherty et al, 1999; New et al, 2004; O'Keane et al, 1992; Virkkunen et al, 1994), and impulsive aggression has been shown to respond the treatment with SSRIs (Coccaro and Kavoussi, 1997). We have recently reported gray matter reduction in anterior cingulate (BA 24) in a large sample of BPD patients $(n=50)$ compared with healthy controls $(n=50)$ (Hazlett et al, 2005). In an analysis of a large sample of BPD-(IED) impulsive aggressive subjects, we found that male subjects with IED-BPD have hypometabolism widely across the frontal lobe compared to healthy men, healthy women and women with BPD in response to placebo (New et al, under review). This finding extends our prior finding of decreased rGMR in response to $\mathrm{m}-\mathrm{CPP}$ in IED-BPD in anterior and increase in posterior cingulate compared to controls in a larger sample (New et al, 2002). We find similar evidence of anterior cingulate decreases and posterior cingulate increases in activation after serotonergic stimulus. We report these findings in a separate manuscript from the present one as they are conceptually quite different. The present study is a correlational analysis, 
examining the relationship of activity in different brain regions, whereas our replications study closely follows the analysis of our previous publication (New et al, 2002), additionally exploring the role of aggression subtype and sex on the findings.

To date, only few neuroimaging studies have evaluated amygdala volume or activity in BPD and none specifically in aggressive subjects. An early study of amygdala volume in BPD showed that total amygdala volume tended to be reduced in female BPD subjects compared to controls (Driessen et al, 2000). Two subsequent studies also reported decreased amygdala volume in BPD compared to controls in relatively small samples (Schmahl et al, 2003; Tebartz van Elst et al, 2003), although a recent study showed no difference in amygdala volume compared to controls (Brambilla et al, 2004) and a VBM extension study showed decreases only in the left hippocampus/amygdala complex (Rusch et al, 2003; Tebartz van Elst et al, 2003). A recent larger study employing a software package 'BRAINS' showed no difference in amygdala volume in BPD compared to controls, although those BPD patients with a concurrent major depressive episode had larger amygdala volumes compared to those without (Zetzsche et al, 2006).

Functional imaging studies of BPD are also limited in number. One study of six female BPD patients and six healthy volunteers (Herpertz et al, 2001) showed that BPD patients had greater cerebral blood flow (BOLD) signal in the amygdala bilaterally during unpleasant pictures compared with neutral pictures than healthy controls. Another study reported greater left amygdala activation in BPD patients to facial expressions of emotion ( $v s$ a fixation point) compared with healthy controls (Donegan et al, 2003).

Taken together, these studies provide support for a model in which the amygdala is linked to emotional processing, but it does not act in isolation; instead functions within a network of brain regions that together modulate the complex manifestations of emotion. This reciprocal interaction predicts that if cortical control of the thalamoamygdala pathway is reduced, emotional responses will be dysregulated (LeDoux, 1994). Based on this literature, we hypothesized that in BPD, an amygdala uncoupled from the prefrontal regulation might be associated with loss of behavioral control. In addition, the numerous data showing abnormalities in serotonergic function in impulsive aggressive patients with personality disorders led us to examine the affect of a serotonergic agent on differential amygdala connectivity with the prefrontal cortex. We further hypothesized that these changes might be more marked for the ventral than dorsal amygdala. Using ${ }^{18}$ FDG-PET, we tested the PFC-amygdala balance theory by comparing interregional correlations between all 13 ipsilateral prefrontal Brodmann areas and amygdala regions in impulsive aggressive BPD patients compared with healthy controls as measured at rest and after a serotonergic stimulus. We predicted more robust correlations between medial OFC (BA 11, 12, and 47) and specifically ipsilateral ventral amygdala in healthy controls compared to patients. We examined ipsilateral correlations as evidence suggests that the reciprocal PFC-amygdala connections both in nonhuman primates (Ghashghaei and Barbas, 2002) and in human beings (Di Virgilio et al, 1999) are predominantly ipsilateral. In addition, we examined group differences in amygdala volume and metabolic activity at baseline and after $\mathrm{m}$-CPP. Data on regional metabolism in PFC and cingulate on a subset of patients included in this study (13 IED-BPD; 13 controls) have been published previously (New et al, 2002).

\section{MATERIALS AND METHODS}

\section{Subjects}

Twenty-six patients (17 men (35.7, SD $=7.9$ years), nine women $(30.7, \mathrm{SD}=8.6)$, range $=20-48 ; 19$ (right-handed), four (left-handed), four (mixed)) meeting DSM-IV criteria for BPD and Intermittent Explosive Disorder-modified (IED) as defined by the Module for Intermittent Explosive disorder (Coccaro et al, 1998) were included. Patients with a history of schizophrenia, a psychotic disorder, or bipolar (Type I) affective disorder were excluded. Patients with current major depressive disorder were excluded. Patients with past or current PTSD were accepted into the study, as a relatively high rate of PTSD in community samples of BPD has been reported (Swartz et al, 1990). Three (all male) of the 26 BPD patients met criteria for current PTSD and one female patient met criteria for past PTSD. We studied borderline patients with impulsive aggression to find a more homogeneous group of subjects with severe symptoms; this resulted, however, in our having a higher portion of male subjects than is usually reported in BPD samples. All subjects were medication-free $>6$ weeks $(22 / 27$ nevermedicated). Twenty-four age- and sex-matched healthy subjects were also studied ( 15 men $(31.7, \mathrm{SD}=7.9$ years); nine women (34.0, $\mathrm{SD}=11.2)$ range $=21-58 ; 19$ (righthanded), two (left-handed), three (mixed)). One RH 31year-old male control was inadequately imaged on the $\mathrm{m}$-CPP day owing to technical difficulties and was used only in baseline analyses. Subjects were screened for severe medical or neurological illness, head injury, or past substance dependence, as well as substance abuse in the prior 6 months. All subjects had a negative urine toxicology screen, and females a negative pregnancy test on each scan day. Participants provided written informed consent in accordance with IRB guidelines. Patients were recruited through advertisement in local newspapers (90\%) and referrals from psychiatric clinics at the Bronx VAMC and Mount Sinai (10\%). For the 26 subjects recruited into the patient group, 164 subjects were screened. Patients were excluded, in order of frequency for not fully meeting BPD, current substance abuse, medical problems, pregnancy, and/or current major depression. One subject declined participation because of the radioactivity and another declined an intravenous line. In the control group, 121 candidates responded to advertisement and 97 were excluded because of the presence of an Axis I or II diagnosis in themselves or a first-degree relative.

Diagnoses were made through interviews by a psychologist using the Structured Clinical Interview for DSM-IV Axis I disorders (First et al, 1996) and the Structured Interview for DSM-IV Personality Disorders (Pfohl et al, 1997), respectively followed by a consensus meeting. Subjects and (when available with the patient's consent) a family member were interviewed. All patients met DSM-IV 
Table I Clinical Assessments in IED-BPD and Controls

\begin{tabular}{|c|c|c|c|c|}
\hline & BDHI* & ALS* & BIS-7B* & CTQ* \\
\hline Controls $(n=22)$ & $17.7, \mathrm{SD}=8.6$ & $0.30, \mathrm{SD}=0.30$ & $36.0, S D=18.3$ & $30.3, S D=12.3$ \\
\hline IED-BPD $(n=24)$ & $40.4, S D=10.1$ & $1.44, \mathrm{SD}=0.58$ & $50.6, S D=14.4$ & $52.5, \mathrm{SD}=20.3$ \\
\hline
\end{tabular}

criteria for BPD, except one subject who met 4/5 criteria needed for a BPD diagnosis by his report and full criteria by family-member report. Trait aggression was assessed using the 'Module for Intermittent Explosive DisorderModified' (Coccaro et al, 1998). All subjects completed the Buss-Durkee Hostility Inventory (BDHI) (Buss and Durkee, 1957), the Barratt Impulsivity Scale (BIS-7b) (Barratt, 1965), the Affective Lability Scale (ALS) (Harvey et al, 1989) and the Childhood Trauma Questionnaire (CTQ) (Bernstein and Fink, 1998).

All BPD patients had: significant physical and/or verbal aggression, meeting criteria for IED $(\kappa=0.92)$. All patients met the 'impulsiveness' criterion for BPD $(\kappa=0.78)$ and $3 / 27$ subjects met the 'self-damaging' BPD criterion $(\kappa=0.90)$. Controls met none of the above-defined criteria. Handedness was determined with the Edinburgh-handedness-scale (Oldfield, 1971). Table 1 shows group means for symptom domains.

\section{Procedure}

On two separate occasions (1-4 weeks apart), each participant received $\mathrm{m}$-CPP or placebo in a double-blind counterbalanced manner. After an overnight fast, an intravenous line was inserted (for blood sampling and injection of $\left.\mathrm{m}-\mathrm{CPP} / \mathrm{placebo} /{ }^{18} \mathrm{FDG}\right)$. $0.08-\mathrm{mg} / \mathrm{kg}$ of $\mathrm{m}-\mathrm{CPP} / \mathrm{placebo}$ was given by slow push, immediately followed by $5 \mathrm{mCi}$ of ${ }^{8}$ FDG. The subject remained resting in a sound-attenuated, dimly-lit room for the 35-min tracer-uptake period. Following uptake, subjects were positioned in the PET scanner for a 45-min data-acquisition period. This method has been described in detail in previous reports (New et al, 2002).

PET scans were carried out as described elsewhere (Haznedar et al, 1997; New et al, 2002) (GE2048 headdedicated scanner, resolution $4.5 \mathrm{~mm}$ in plane, $5.0 \mathrm{~mm}$ axially). Fifteen slices at $6.5-\mathrm{mm}$ intervals were obtained in two sets to cover the entire brain. Slice counts of $1.5-3 \mathrm{M}$ counts are typical. Scans were reconstructed with a blank and a transmission scan using the Hanning filter. The same individually molded thermoplastic facemask was used for each scan to minimize head-movement during image acquisition and to assist in PET/MRI coregistration. PET images were obtained in nanocuries/pixel and standardized as rGMR by dividing each pixel by the mean value for the entire brain (defined by brain-edge from coregistered MRI). Although this limits interpretations of single structure absolute activity, this method is widely used when evaluating hypotheses related to patterns of metabolic rate across brain areas and was used in earlier imaging studies of serotonin activation (Mann et al, 1996; New et al, 2002;
Siever et al, 1999a; Soloff et al, 2000). PET-MRI coregistration used the algorithm of Woods et al (1993). Brain edges were visually traced on all MRI axial slices with inter-tracer reliability of 0.99 on 10 subjects.

\section{Regions of Interest Approach}

We assessed rGMR within BAs by tracing coronal slices based on a digitized brain atlas with 33 coronal slice maps of BAs defined by microscopic examination of an entire postmortem brain, a technique detailed elsewhere (Buchsbaum et al, 2001, 2002; Hazlett et al, 2000; Mitelman et al, 2005). To assess the effect of $\mathrm{m}-\mathrm{CPP}$ on rGMR, the dependent measure for PET analyses on drug effect was expressed as difference scores (m-CPP-placebo) for rGMR within each BA, calculated by subtracting placebo counts for each region of interest in each subject from the corresponding rGMR from the $\mathrm{m}-\mathrm{CPP}$ scan.

The amygdala was outlined on coronal MRI sections using previously published methods (Haznedar et al, 2000) (ICC $=0.82$, area measured on three slices at the 25th, 50th, and 75th percentiles of anteroposterior distance). Outlining of the amygdala began at its largest extent (approximately the center in the anteroposterior dimension) where clear boundaries between gray matter and surrounding white matter are visible. At this mid-section, the amygdaloid complex is roughly elliptical in shape, and anatomical margins are defined by the cornu ammonis and the white matter of gyrus ambiens in the medial aspect, the cornu inferius of the lateral ventricle in the ventral aspect, the temporal lobe white matter laterally, and the gyrus semilunaris in the dorsal aspect. Using an edge contrastenhancing technique (gradient filter) (Haznedar et al, 2000), we were able to visualize better the dentate gyrus of the hippocampus and boundaries between the hippocampus and the amygdala. The posterior portions of the amygdaloid complex were outlined by using the ventricular recess, hippocampus, and gyrus semilunaris as reference points (Figure 1). Anteriorly, the amygdaloid complex gray matter is more heterogeneous and hard to identify. We outlined from the midsection forward using gradient filtering and excluded the entorhinal cortex, which may include the inferior amygdala. The outlining ended at the first coronal MRI section on which there was visible white matter between the amygdala, ambiens, and white matter of the entorhinal cortex. This procedure may have omitted the very anterior end of the amygdaloid complex, but it had the advantage of excluding other extraneous structures from our analysis. Following the suggestion of Kim et al (2003) we divided the amygdala into a top and bottom half, based on the vertical distance on the mid-coronal slice and 


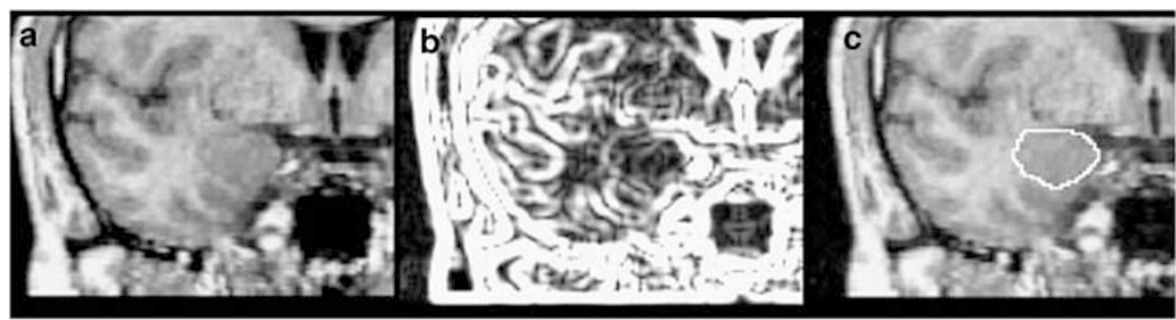

Figure I Tracing the amygdala. (a) Coronal MRI: anterior-posterior dimension of amygdala. (b) Sobel-gradient filter to enhance gray/white boundaries. (c) Amygdala outlined.

applied the MRI-traced template to resliced and coregistered coronal PET slices. Volume is expressed as absolute volume in $\mathrm{mm}^{3}$ and relative to whole brain volume. The data were assessed for movement artifact by measuring the ratio of the area of the middle PET slice (obtained by a radial edging algorithm which draws the edge at $62 \%$ of the maximum value) to the area of the middle MRI slice (obtained by hand-tracing); head-movement during the longer scan would tend to blur the image and enlarge the area of activity. No group difference for movement artifact was detected (normals $=0.967 \pm 0.031$, patients $=$ $0.964 \pm 0.037, t=0.30)$.

\section{Statistical Analysis}

Pearson's correlation coefficients were employed for frontal BAs and regions of the amygdala with significance level of $p<0.05$. The Kullback's $\chi^{2}$ test for correlational matrices was employed to test group differences in correlational matrices (Kullback, 1967). The Kullback test provides one single $p$-value for comparing two correlation matrices each from a separate group of subjects. This avoids the Type I error associated with multiple correlation testing because only one comparison is made. Univariate tests can then be supplied as post hoc tests to locate major sources of matrix differences. Mixed-factorial repeated-measures ANOVAs were employed to examine group differences in amygdala volume and metabolism. In addition, Pearson's correlation coefficients were used to test correlation between amygdala activity and the ALS, BIS, BDHI and CTQ scores. All correlations are reported for $p<0.05$, two-tailed.

ANOVAs examining amygdala volume and function were conducted both including and excluding the subjects meeting PTSD, as PTSD has been associated with both increased (Protopopescu et al, 2005; Shin et al, 2004), and decreased amygdala activity (Britton et al, 2005); amygdala volume in PTSD has been shown not to be different from controls (Bremner, 2002; Wignall et al, 2004).

\section{RESULTS}

\section{Correlations PFC and Amygdala}

Placebo condition: correlations PFC and amygdala. The correlations between the ventral amygdala and orbitofrontal BA 11, BA 12, and BA 47 were positive and significant in controls on the right; whereas on the left, they were positive but not significant in BA 11, BA 12, and BA 47 (Table 2a). In contrast, the IED-BPD group showed no significant correlations between right or left ventral amygdala and OFC (BA 11, 12, 47) (Table 2a). In addition to OFC, controls showed positive correlations between subgenual BA 25 and ventral amygdala on the right and between BA 44 and ventral amygdala bilaterally. Controls showed no significant positive correlations between any frontal BAs and dorsal amygdala in either hemisphere. Patients showed no significant positive correlations between frontal BAs and either dorsal or ventral amygdala at rest. In patients, there were significant negative correlations between the dorsal and ventral amygdala and $B A s 6,8,9,10,32$, and 46 bilaterally.

To test for group differences in the correlation matrices for ventral/dorsal amygdala with frontal BAs for each hemisphere, a Kullback's $\chi^{2}$ test was conducted (Kullback, 1967). Controls differed significantly from patients in correlations between prefrontal BAs and dorsal and ventral amygdala in both hemispheres (right ventral amygdala: $\mathrm{df}=91$, Kullback's $\chi^{2}=236.853, p<0.0001$; right dorsal amygdala: $\mathrm{df}=91$, Kullback's $\chi^{2}=193.939, \quad p<0.001$; left ventral amygdala, $\mathrm{df}=91$, Kullback's $\chi^{2}=194.290, p<0.001$; left dorsal amygdala, $\mathrm{df}=91$, Kullback's $\chi^{2}=184.355, p<0.001$ ) (see Figure 2). The most striking group differences occurred in correlations between right ventral amygdala and orbital BAs 11, 12, and 47. Figure 3 shows scatter plots of rGMR for correlations between these orbital BAs in the right hemisphere and ventral amygdala.

$m$-CPP-placebo condition: correlations PFC and amygdala. In response to $\mathrm{m}-\mathrm{CPP}$, controls showed significant positive correlations between ventral amygdala and BA 12 and 25 on the left. In addition, controls showed significant positive correlations for $\mathrm{m}$-CPP-placebo rGMR between dorsal amygdala and left BA 11 and 12 and right BA 11 and 25. In controls, the frontal pole correlated negatively with dorsal amygdala in response to $\mathrm{m}-\mathrm{CPP}$ in right BA 6 and 8 (see Table 2b). Patients, in contrast had positive correlations between dorsal amygdala and dorsolateral PFC BA 44,45 and 46 on the left and between ventral amygdala and dorsolateral PFC in BA 44 and 45. In addition, patients showed a positive correlation between BA 12 and left ventral amygdala and a negative correlation between dorsal amygdala and BA 8 on the left.

Also for m-CPP-placebo, controls differed significantly from patients in correlations between the prefrontal BAs and ipsilateral dorsal and ventral amygdala in both hemispheres (right ventral amygdala: $\mathrm{df}=91$, Kullback's $\chi^{2}=190.04, p<0.001$; right dorsal amygdala: $\mathrm{df}=91$, Kullback's $\chi^{2}=248.71, p<0.001$; left ventral amygdala, $\mathrm{df}=91$, 
Table 2 Correlations Frontal Brodmann Areas and Amygdala: Placebo and m-CPP-Placebo Condition

\begin{tabular}{|c|c|c|c|c|c|c|c|c|c|c|c|c|c|}
\hline & BA6 & BA8 & BA9 & BAIO & BAII & BAI 2 & BA24 & BA25 & BA32 & BA44 & BA45 & BA46 & BA47 \\
\hline \multicolumn{14}{|l|}{ (a) Placebo } \\
\hline \multicolumn{14}{|l|}{ Control } \\
\hline Right dorsal amygdala & $-0.43 *$ & -0.34 & -0.31 & $0.02^{\star}$ & $0.39^{\star}$ & $0.39^{\star}$ & -0.08 & 0.34 & $0.07^{\star}$ & 0.27 & 0.22 & $0.12^{\star}$ & 0.37 \\
\hline Left dorsal amygdala & -0.17 & -0.20 & -0.15 & $-0.03^{\star}$ & 0.07 & 0.17 & -0.24 & 0.01 & $-0.27^{\star}$ & $0.35^{\star}$ & 0.24 & -0.11 & 0.17 \\
\hline Right ventral amygdala & $-0.46 *$ & -0.19 & -0.20 & 0.11 & $0.46 *$ & $0.54^{*}$ & -0.09 & $0.44 *$ & $0.19^{\star}$ & $0.50 *$ & 0.32 & 0.09 & $0.49 *$ \\
\hline \multicolumn{14}{|l|}{$B P D$} \\
\hline Right dorsal amygdala & $-0.50 *$ & $-0.55 *$ & $-0.57 *$ & $-0.56 *$ & -0.26 & -0.28 & -0.20 & -0.24 & $-0.63 *$ & -0.29 & -0.28 & $-0.52 *$ & -0.15 \\
\hline Left dorsal amygdala & $-0.62 *$ & $-0.59 *$ & $-0.60 *$ & $-0.63 *$ & -0.29 & -0.20 & -0.21 & -0.33 & $-0.58 *$ & -0.36 & -0.25 & $-0.58 *$ & -0.32 \\
\hline Right ventral amygdala & $-0.6 I^{*}$ & $-0.62 *$ & $-0.59 *$ & $-0.41 *$ & -0.07 & -0.02 & -0.32 & -0.03 & $-0.61 *$ & -0.31 & -0.28 & $-0.53 *$ & 0.02 \\
\hline Right dorsal amygdala & $-0.57 *$ & $-0.55 *$ & -0.33 & -0.28 & $0.42 *$ & $0.40^{\star}$ & -0.19 & $0.44^{*}$ & 0.16 & 0.08 & 0.27 & -0.10 & 0.35 \\
\hline Left dorsal amygdala & -0.17 & $-0.45 *$ & -0.02 & -0.06 & $0.45 *$ & $0.49 *$ & -0.08 & 0.32 & -0.25 & 0.25 & 0.31 & 0.03 & 0.39 \\
\hline Right ventral amygdala & -0.29 & -0.21 & -0.15 & $0.30^{\star}$ & 0.21 & 0.21 & -0.36 & 0.36 & -0.02 & 0.18 & 0.07 & -0.22 & 0.28 \\
\hline Left ventral amygdala & -0.11 & -0.17 & -0.03 & -0.09 & 0.37 & $0.44 *$ & -0.18 & $0.43 *$ & 0.16 & 0.36 & 0.18 & -0.05 & 0.28 \\
\hline \multicolumn{14}{|l|}{ BPD } \\
\hline Right dorsal amygdala & $0.05^{\star}$ & $0.03^{\star}$ & -0.15 & -0.18 & -0.35 & -0.32 & -0.13 & $-0.37^{\star}$ & -0.18 & -0.28 & -0.21 & -0.19 & -0.23 \\
\hline Left dorsal amygdala & -0.38 & $-0.45^{*}$ & -0.28 & -0.22 & 0.23 & 0.43 & -0.01 & 0.10 & -0.13 & $0.44 *$ & $0.62 *$ & $0.20 *$ & 0.15 \\
\hline Right ventral amygdala & -0.21 & -0.25 & -0.36 & -0.31 & -0.28 & -0.16 & -0.17 & 0.36 & -0.22 & -0.36 & -0.09 & -0.26 & -0.27 \\
\hline Left ventral amygdala & -0.12 & -0.24 & -0.03 & 0.03 & 0.30 & $0.40 *$ & -0.16 & 0.26 & -0.02 & $0.54 *$ & $0.54^{*}$ & 0.36 & 0.28 \\
\hline
\end{tabular}

Relative glucose metabolism (rGMR) correlations between frontal lobe brodmann areas and dorsoventral amygdala divisions for placebo (a) and m-CPP-placebo (b). Asterisks denote significant Pearson correlation coefficients $(p<0.05)$. Diamonds denote significantly different correlations for patient group from control group for a particular region of interest, ( $p<0.05$, two-sided).

Red demonstrates significant positive correlation coefficients between indicated brain regions, whereas bold indicates significantly negative correlation coefficients between brain regions.
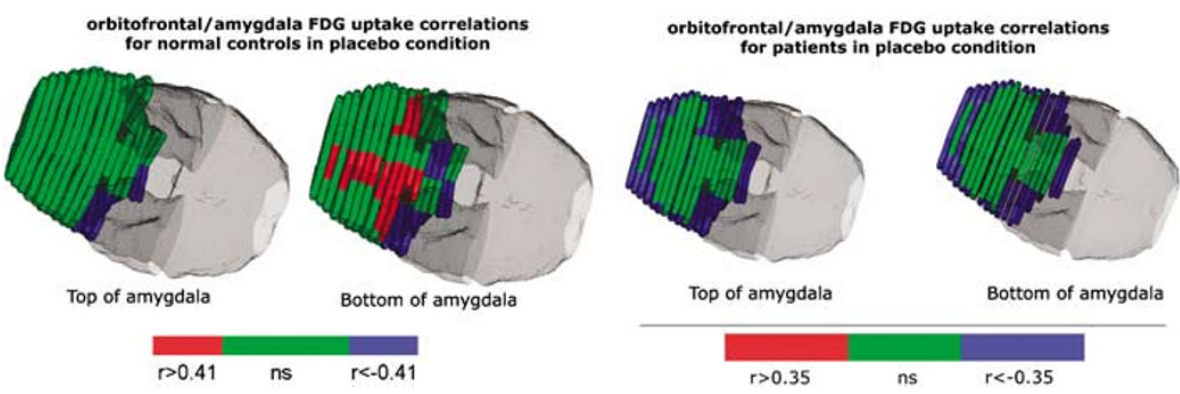

Figure 2 Correlations orbital floor and amygdala: placebo condition, This is a visual map of significant positive and negative as well as non-significant correlations between rGMR in frontal Brodmann areas on the orbital surface of the brain and rGMR in ipsilateral amygdala in healthy subjects on the left and in impulsive aggressive borderline personality disorder subjects on the right. Significant positive correlations at $p<0.05$ are shown in red and significant negative correlations in blue.

Kullback's $\chi^{2}=169.10, p<0.001$; left dorsal amygdala, $\mathrm{df}=91$, Kullback's $\chi^{2}=194.40, p<0.001$ ) (see Figure 4 ).

\section{Group Differences in Amygdala Volume and Metabolism}

Although our hypothesis in this study was that the coupling of metabolic activity between frontal BAs and ventral amygdala would be disrupted, we also tested simple group differences in amygdala volume and metabolism.

Amygdala volume. Because of evidence for specificity of ventral amygdala in human emotion, we divided the amygdala into dorsal and ventral components. A group (control, patient) $\times$ hemisphere $(\mathrm{R}, \mathrm{L}) \times$ region (dorsal, ventral) repeated-measures ANOVA with dorsal and ventral 

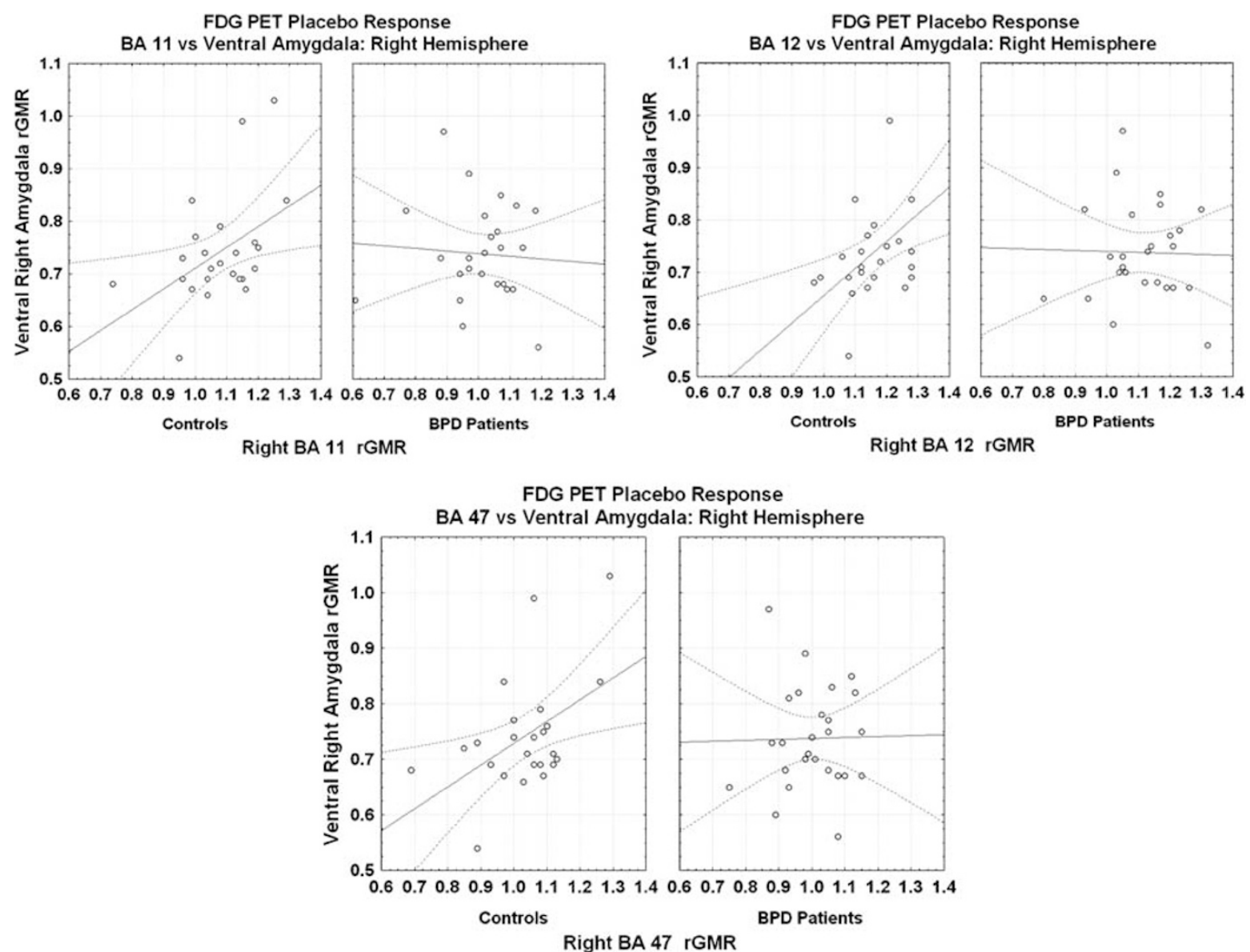

Figure 3 Scatterplots right hemisphere OFC/ventral amygdala. Scatterplots of correlations of orbital Brodmann areas and amygdala rGMR for right BA I I, 12, 47 and ventral amygdala illustrating distribution in controls and in IED-BPD.
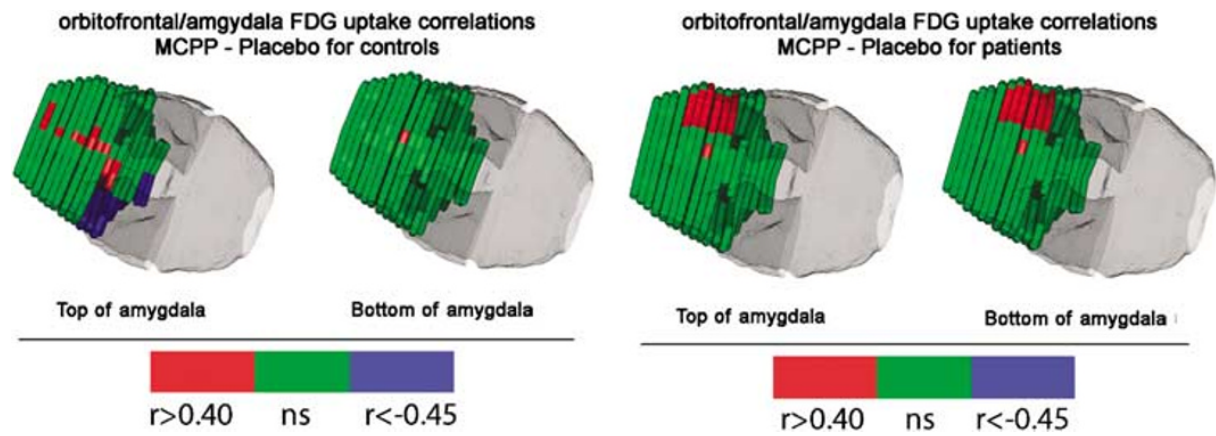

Figure 4 Correlations orbital floor and amygdala: $m$-CPP- Placebo Condition. This is a visual map of significant positive and negative as well as nonsignificant correlations between rGMR for m-CPP minus placebo in frontal Brodmann areas on the orbital surface of the brain and rGMR for m-CPP minus placebo in ipsilateral amygdala in healthy subjects on the left and in impulsive aggressive borderline personality disorder subjects on the right. Significant positive correlations at $p<0.05$ are shown in red and significant negative correlations in blue.

amygdala volume as the dependent variable showed no main effect of group or interaction with group. The same result was found with relative amygdala volume (amygdala volume/whole brain volume). It should be noted that the total amygdala volumes found in our healthy subjects fall well within the range of that found by others. Specifically, we found total amygdala volumes for healthy control subjects (mathematically identical of the sum of the top and bottom for each hemisphere) to be: right hemisphere:
$1365 \pm 198 \mathrm{~mm}^{3}$ for women, $1397 \pm 208 \mathrm{~mm}^{3}$ for men; left hemisphere: $1198 \pm 254 \mathrm{~mm}^{3}$ for women, $1365 \pm 225 \mathrm{~mm}^{3}$ for men. This is within the range for normal human amygdala volumes found by others ranging from 1050 to $1600 \mathrm{~mm}^{3}$ for the right amygdala and 1140 to $1400 \mathrm{~mm}^{3}$ for the left amygdala (Convit et al, 1999; Driessen et al, 2000; Szabo et al, 2003).

Similarly, when the same analysis was conducted excluding subjects meeting criteria for PTSD, no significant effect 
Table 3 Amygdala Volume

\begin{tabular}{|c|c|c|}
\hline Absolute volume $\mathrm{mm}^{3}$ (SD) & Controls $(n=23)$ & IED-BPD $(n=26)$ \\
\hline Right dorsal amygdala & $919(155)$ & $889(|4|)$ \\
\hline Left dorsal amygdala & $888(158)$ & $861(153)$ \\
\hline Right ventral amygdala & $1004(\mid 40)$ & $926(149)$ \\
\hline Left ventral amygdala & $956(162)$ & $927(194)$ \\
\hline \multicolumn{3}{|c|}{ Relative to whole brain $\times 1000$, mean, SD } \\
\hline Right dorsal amygdala & $0.11(0.02)$ & $0.10(0.02)$ \\
\hline Left dorsal amygdala & $0.10(0.01)$ & $0.10(0.02)$ \\
\hline Right ventral amygdala & $0.12(0.02)$ & $0.11(0.02)$ \\
\hline Left ventral amygdala & $0.11(0.01)$ & $0.11(0.02)$ \\
\hline
\end{tabular}

Absolute volume in $\mathrm{mm}^{3}$ and relative to whole brain volume for dorsoventral amygdala divisions.

of group in relative volume was detected. The groups did not differ in whole brain volume (controls mean 1190, $\mathrm{SD}=150 \mathrm{~mm}^{3}$, patients $1180, \quad \mathrm{SD}=125 \mathrm{~mm}^{3}, \quad t=0.04$, $p=$ NS) (see Table 3).

Amygdala metabolism: placebo. A group (control, patient $) \times$ hemisphere $(\mathrm{R}, \mathrm{L}) \times$ region $($ dorsal, ventral) repeated-measures ANOVA with placebo rGMR in the dorsal/ventral amygdala as the dependent variable showed no main effect of group or significant interaction with group. Similarly, there was no interaction involving group for rGMR in the whole amygdala. The same result was found when excluding the subjects with PTSD.

Amygdala metabolism (m-CPP-placebo). A group (control, patient $) \times$ hemisphere $(\mathrm{R}, \mathrm{L}) \times$ region (dorsal,ventral) repeated-measures ANOVA with rGMR $\mathrm{m}$-CPP-placebo difference scores within regions of the amygdala as the dependent variable showed no interaction involving group. The same result was found when excluding the subjects with PTSD.

Correlations of volume/metabolism with clinical symptoms. To examine the relationship between metabolism and symptom dimensions, we examined correlations between volume and metabolism and clinical measures of impulsivity, aggression, and affective instability.

Amygdala volume. We observed a negative correlation between BIS score and right relative ventral amygdala volume in controls $(n=22, r=-0.45, p<0.001)$, but not patients ( $n=23, r=-0.27, p=\mathrm{NS}$ ) (between-group test of correlation differences was not significant). In patients, we found a negative correlation between impulsivity scores and left ventral amygdala volume $(n=23, r=-0.42, p<0.004)$, not present in controls $(n=22 r=-0.08, p=$ NS) (betweengroup test of correlation differences was not significant). These correlations survive Bonferroni correction for multiple comparisons for which a $p$-value $<0.004$, two-tailed, is significant.
Amygdala activity. In patients, but not controls, we observed a negative correlation between aggression and affective lability scores for placebo rGMR in right ventral and dorsal amygdala (BDHI: ventral, $r=-0.67$, dorsal $r=0.63$; ALS: ventral, $r=-0.67$, dorsal $r=-0.68)$. For drug-placebo, in controls but not patients, we observed negative correlations between ALS scores and the ventral amygdala (controls $r=-0.64$ ) (Bonferroni corrected significance, $p<0.002$, two-tailed) (between group test of correlation differences was not significant).

\section{DISCUSSION \\ Implications of Group Differences in Fronto-Amygdala Correlations}

The most striking finding that we report is the highly significant normal-BPD group differences in correlation patterns between frontal BA and ipsilateral amygdala metabolic activity (m-CPP minus placebo as well as the resting condition). Healthy controls, both at rest and in response to a serotonergic probe, show positive correlations between $\mathrm{OFC}(\mathrm{BA} 11,12)$ and right ventral amygdala, as was predicted from the model of the ventral amygdala as the component of human amygdala most closely associated with frontal lobe emotion modulation (Kim et al, 2003; Somerville et al, 2004; Whalen et al, 1998). This correlational approach is based on the assumption that significant correlations may reveal an important functional relationship between the structures as discussed by Katz et al (1996). Correlations in healthy controls support the idea of intact coupling between $\mathrm{PFC}$, particularly the ventrolateral region (BA 11, 47 and more dorsally in BA 44), and right ventral amygdala, a tight coupling which may be the neural substrate for downregulation of the amygdala in response to aversive stimuli. The absence of such tight coupling in BPD patients, indicated by the lack of significant correlations between OFC and amygdala, suggests a disconnect between OFC and amygdala, which may explain the failure of BPD patients to downregulate the amygdala in response to aversive stimuli. Furthermore, the BPD patients appear to have lost anatomic specificity of ventral $v s$ dorsal amygdala, showing nonsignificant correlations with OFC and modest negative correlations between amygdala and widespread regions of the frontal lobe.

The directionality of correlations between amygdala and PFC is inconsistent in the literature. Our studies are consistent with findings of Pezawas et al, in which rostral areas of anterior cingulate showed positive correlations with amygdala, at least under certain conditions (Kim et al, 2004; Pezawas et al, 2005). However, other studies have demonstrated negative correlations between amygdala and medial OFC (Hariri et al, 2000, 2003). Previous fMRI studies showing negative correlations between amygdala and OFC have employed event-related designs showing acute reactions to aversive stimuli (Dougherty et al, 2004; Shin et al, 2005). Our study, in contrast, employs ${ }^{18}$ FDG-PET, which reflects an average of activity of a 30-min epoch. As FDG uptake appears to reflect metabolic activity at axon terminals (Sokoloff, 1982), positive correlations would arise between areas that receive input causing firing and its area of efferent connection (Katz et al, 1996). A limitation of the 
correlational approach using FDG is that it does not discriminate between inhibitory and excitatory connections.

Another reason for inconsistencies in the literature arise from the specific brain regions examined. Findings of a negative correlation between the regions of PFC and amygdala have been found with the rostral anterior cingulate (Pezawas et al, 2005), as well as in ventral regions of PFC (Hariri et al, 2000, 2003). Our finding of positive correlations between particularly lateral OFC and ventral amygdala in healthy subjects (not found in our borderline group) is consistent with regional specificity of the connection between amygdala and PFC shown in primate studies (Carmichael and Price, 1995), and some human studies however (Kim et al, 2004).

The ventral amygdala has been suggested to have greater interconnection with OFC whereas the dorsal amygdala has greater interconnection with the cingulate and other limbic areas (Aggleton and Saunders, 2000). In our data, cingulate areas left 24 and right 25 were both significantly correlated with ventral but not dorsal amygdala, consistent with the general pattern of greater ventral amygdala-cortical links, but not with a distinction between cingulate and OFC connections with the amygdala.

We show no significant group differences in rGMR between BPD patients and controls in the amygdala, which may suggest that the primary abnormality in BPD relates to the failure of the PFC to 'come on line' in response to amygdala activation. Group differences in correlations in response to serotonergic stimulation show healthy controls with positive correlations between orbitofrontal areas (BA 11, bilaterally; left BA 12) and dorsal amygdala (Table 2b). This is consistent with evidence that the dorsal amygdala (central nucleus) is rich in neuronal fibers positive for serotonin and serotonin-transporter in primates (Freedman and Shi, 2001). Borderline patients show positive correlations between dorsal and ventral amygdala and dorsolateral BAs.

\section{Implications of Lack of Group Differences in Amygdala Volume and Activity}

Consistent with larger studies in the literature employing 'BRAINS' to delineate the amygdala, our hand-tracing of amygdala in a large sample show no difference in amygdala volume between BPD and controls. Although some studies have shown group differences, these studies have employed exclusively female subjects, where as we include both men and women and have selected BPD subjects with impulsive aggression. We explored our data to examine whether entering sex into the ANOVA would yield significant results and found no main effect of sex or interaction between sex and diagnosis in amygdala volume.

Clinical correlation with volume show some laterality in that inverse correlations were seen in patients between right ventral amygdala and measures of impulsivity, but in left ventral amygdala in controls. Prior studies of amygdala volume decreases in BPD, however, have shown a bilateral effect. Clinical correlations in amygdala activity revealed inverse correlations with affective instability and aggression with right ventral and dorsal amygdala in $\mathrm{BPD}$, whereas in controls, a negative correlation between right ventral amygdala and affective lability was observed. This supports our evidence that there is a loss of anatomic specificity between the ventral and dorsal amygdala in BPD. There was no relationship between aggression scores and left ventral amygdala volume in controls in part because the variance in aggression measures within the controls group was very limited.

This study has a number of limitations in design and analysis. The lack of group differences in activation in amygdala may relate to the study design, which did not employ a behavioral task. As there was a pharmacological challenge, the interaction with an impulse control task would have required four scans instead of two, and this would have provided logistical and subject volunteerism problems. The responsivity of the amygdala may not be adequately probed with a resting scan or with a pharmacologic challenge. Future studies involving behavioral provocation during functional scanning will elucidate this. In addition, our statistical analysis employed a broad assessment of correlations between all prefrontal Brodmann areas in each hemisphere as well as the ventral and dorsal amygdala. However, as functional imaging studies, present and future, are producing a large number of activation areas from exploratory mapping, the full presentation of the correlation matrix seemed justified to provide findings for other exploratory imaging studies.

Another limitation of the present study is the lack of precision of our top-bottom parcellation of the amygdala. The amygdala ROI has a volume of about $1300 \mathrm{~mm}^{3}$ in our tracings. The full-width half-maximum resolution of our scanner measured at the center of the ring is $4.5 \mathrm{~mm}$ in plane and $6.5 \mathrm{~mm}$ in the $z$-axis. This yields a volume of $131 \mathrm{~mm}^{3}$ and the amygdala volume is thus approximately 10 times as great. The mean height of our amygdala tracing on the coronal ROI is $14.3 \mathrm{~mm}$, and encompasses two PET slices. Nevertheless, top and bottom amygdala values are one-half as high, and partial volume effects limit our statistical power to detect differences. In addition, our division into top and bottom half is geometrical and does not weight the lateral basal portion. MRI images provide inadequate resolution to easily trace individual amygdala nuclei, again reducing statistical power.

\section{ACKNOWLEDGEMENTS}

This research was supported by NIMH Grants MH566606 to Dr Siever, MH067918 to Dr New, and MH60023 to Dr Buchsbaum, and by the VA Medical Research Program (Career Development Award) to Dr New and a NARSAD Independent Investigator Award to Dr Hazlett. This work was also supported in part by a grant (5-M01 RR00071) for the Mount Sinai General Clinical Research Center from the National Center for Research Resources, at the NIH.

\section{REFERENCES}

Aggleton JP, Saunders RC (2000). The amygdala-what's happened in the last decade? In: Aggleton JP (ed). The Amygdala. A Functional Analysis. Oxford University Press: Oxford. pp 1-30. al Maskati HA, Zbrozyna AW (1989). Cardiovascular and motor components of the defence reaction elicited in rats by electrical and chemical stimulation in amygdala. J Auton Nerv Syst 28: $127-131$. 
Barratt ES (1965). Factor analysis of some psychometric measures of impulsiveness and anxiety. Psychol Rep 16: 547-554.

Bernstein D, Fink L (1998). Childhood Trauma Questionnaire: A Retrospective Self-report. Psychological Corp: San Antonio, TX.

Brambilla P, Soloff PH, Sala M, Nicoletti MA, Keshavan MS, Soares JC (2004). Anatomical MRI study of borderline personality disorder patients. Psychiatry Res 131: 125-133.

Bremner JD (2002). Neuroimaging studies in post-traumatic stress disorder. Curr Psychiatry Rep 4: 254-263.

Britton JC, Phan KL, Taylor SF, Fig LM, Liberzon I (2005). Corticolimbic blood flow in posttraumatic stress disorder during script-driven imagery. Biol Psychiatry 57: 832-840.

Buchsbaum MS, Hollander E, Haznedar MM, Tang C, SpiegelCohen J, Wei TC et al (2001). Effect of fluoxetine on regional cerebral metabolism in autistic spectrum disorders: a pilot study. Int J Neuropsychopharmacol 4: 119-125.

Buchsbaum MS, Nenadic I, Hazlett E, Spiegel-Cohen J, Fleischman MB, Akhavan A et al (2002). Differential metabolic rates in prefrontal and temporal Brodmann areas in schizophrenia and schizotypal personality disorder. Schizophr Res 54: 141-150.

Buss AH, Durkee A (1957). An inventory for assessing different kinds of hostility. J Consult Psychol 21: 343-348.

Carmichael ST, Price JL (1995). Limbic connections of the orbital and medial prefrontal cortex in macaque monkeys. J Comp Neurol 363: 615-641.

Coccaro EF (1989). Central serotonin and impulsive aggression. Br J Psychiatry 155: 52-62.

Coccaro EF, Kavoussi RJ, Berman M, Lish J (1998). Intermittent explosive disorder-revised: development, reliability, and validity of research criteria. Comp Psychiatry 39: 368-376.

Coccaro EF, Kavoussi RJ (1997). Fluoxetine and impulsive aggressive behavior in personality disordered subjects. Arch Gen Psychiatry 54: 1081-1088.

Convit A, McHugh P, Wolf OT, de Leon MJ, Bobinski M, De Santi S et al (1999). MRI volume of the amygdala: a reliable method allowing separation from the hippocampal formation. Psychiatry Res 90: 113-123.

De La Fuente JM, Goldman S, Stanus E, Vizuete C, Morlan I, Bobes $\mathrm{J}$ et al (1997). Brain glucose metabolism in borderline personality disorder. J Psychiatr Res 31: 531-541.

Di Virgilio G, Clarke S, Pizzolato G, Schaffner T (1999). Cortical regions contributing to the anterior commissure in man. Exp Brain Res 124: 1-7.

Dias R, Robbins TW, Roberts AC (1996). Dissociation in prefrontal cortex of affective and attentional shifts. Nature 380: 69-72.

Donegan NH, Sanislow CA, Blumberg HP, Fulbright RK, Lacadie C, Skudlarski P et al (2003). Amygdala hyperreactivity in borderline personality disorder: implications for emotional dysregulation. Biol Psychiatry 54: 1284-1293.

Dougherty DD, Rauch SL, Deckersbach T, Marci C, Loh R, Shin LM et al (2004). Ventromedial prefrontal cortex and amygdala dysfunction during an anger induction positron emission tomography study in patients with major depressive disorder with anger attacks. Arch Gen Psychiatry 61: 795-804.

Dougherty DM, Bjork JM, Huckabee H, Moeller F, Swann A (1999). Laboratory measures of aggression and impulsivity in women with borderline personality disorder. Psychiatry Res 85: 315-326.

Drevets WC, Videen TO, Price JL, Preskorn SH, Carmichael ST, Raichle ME (1992). A functional anatomical study of unipolar depression. J Neurosci 12: 3628-3641.

Driessen M, Herrmann J, Stahl K, Zwaan M, Meier S, Hill A et al (2000). Magnetic resonance imaging volumes of the hippocampus and the amgydala in women with borderline personality disorder and early traumatization. Arch Gen Psychiatry 57: $1115-1122$

Emery N, Capitanio J, Mason W, Machado C, Mendoza S, Amaral D (2001). The effects of bilateral lesions of the amygdala on dyadic social interactions in rhesus monkeys (Macaca mulatta). Behav Neurosci 115: 515-544.

First M, Spitzer R, Gibbon M, Williams J (1996). Structured Clinical Interview for Axis I Disorders-Patient Edition. New York Biometrics Research, New York State Psychiatric Institute: New York, NY.

Freedman LJ, Shi C (2001). Monoaminergic innervation of the macaque extended amygdala. Neuroscience 104: 1067-1084.

Ghashghaei HT, Barbas H (2002). Pathways for emotion: interactions of prefrontal and anterior temporal pathways in the amygdala of the rhesus monkey. Neuroscience 115: 1261-1279.

Gregg TR, Siegel A (2001). Brain structures and neurotransmitters regulating aggression in cats: implications for human aggression. Prog Neuropsychopharmacol Biol Psychiatry 25: 91-140.

Halasz J, Liposits Z, Meelis W, Kruk MR, Haller J (2002). Hypothalamic attack area-mediated activation of the forebrain in aggression. Neuroreport 13: 1267-1270.

Hariri AR, Bookheimer SY, Mazziotta JC (2000). Modulating emotional responses: effects of a neocortical network on the limbic system. Neuroreport 11: 43-48.

Hariri AR, Drabant EM, Weinberger DR (2006). Imaging genetics: perspectives from studies of genetically driven variation in serotonin function and corticolimbic affective processing. Biol Psychiatry 59: 888-897.

Hariri AR, Mattay VS, Tessitore A, Fera F, Weinberger DR (2003). Neocortical modulation of the amygdala response to fearful stimuli. Biol Psychiatry 53: 494-501.

Harvey P, Greenberg B, Serper M (1989). The affective lability scales: development reliability and validity. J Clin Psychol 45: 786-793.

Hazlett E, Buchsbaum M, Jeu L, Nenadic I, Fleischman MB, Shihabuddin L et al (2000). Hyporfrontality in unmedicated schizophrenia patients studied with PET during performance of a serial verbal learning task. Schizophr Res 43: 33-46.

Hazlett EA, New AS, Newmark R, Haznedar MM, Lo JN, Speiser LJ et al (2005). Reduced anterior and posterior cingulate gray matter in borderline personality disorder. Biol Psychiatry 58: 614-623.

Haznedar M, Buchsbaum M, Metzer M, Solimando A, SpeigelCohen J, Hollander E (1997). Anterior cingulate gyrus volume in glucose metabolism in autistic disorder. Am J Psychiatry 154: 1043-1045.

Haznedar M, Buchsbaum M, Wei T, Hof PR, Cartwright C, Bienstock C et al (2000). Limbic circuitry in patients with autism spectrum disorders studied with positron emission tomography and magnetic resonance imaging. Am J Psychiatry 157: 1994-2001.

Heinz A, Braus DF, Smolka MN, Wrase J, Puls I, Hermann D et al (2005). Amygdala-prefrontal coupling depends on a genetic variation of the serotonin transporter. Nat Neurosci 8: 20-21.

Herpertz S, Dietrich T, Wenning B, Krings T, Erberich S, Willmes $\mathrm{K}$ et al (2001). Evidence of abnormal amygdala functioning in borderline personality disorder: a functional MRI study. Biol Psychiatry 50: 292-298.

Izquierdo A, Suda RK, Murray EA (2005). Comparison of the effects of bilateral orbital prefrontal cortex lesions and amygdala lesions on emotional responses in rhesus monkeys. J Neurosci 25: 8534-8542.

Jinks AL, McGregor IS (1997). Modulation of anxiety-related behaviours following lesions of the prelimbic or infralimbic cortex in the rat. Brain Res 772: 181-190.

Katz M, Buchsbaum MS, Siegel Jr BV, Wu J, Haier RJ, Bunney Jr WE (1996). Correlational patterns of cerebral glucose metabolism in never-medicated schizophrenics. Neuropsychobiology 33: $1-11$.

Kim H, Somerville LH, Johnstone T, Alexander AL, Whalen PJ (2003). Inverse amygdala and medial prefrontal cortex responses to surprised faces. Neuroreport 14: 2317-2322. 
Kim H, Somerville LH, Johnstone T, Polis S, Alexander AL, Shin LM et al (2004). Contextual modulation of amygdala responsivity to surprised faces. J Cogn Neurosci 16: 1730-1745.

Kullback S (1967). On testing correlational matrices. Appl Statist 16: $80-85$.

LeDoux JE (1994). Emotion, memory and the brain. Sci Am 270: 50-57.

Mann JJ, Malone KM, Diehl DJ, Perel J, Nichols TE, Mintun MA (1996). Positron emission tomographic imaging of serotonin activation effects on prefrontal cortex in healthy volunteers. J Cereb Blood Flow Metab 16: 418-426.

McDonald AJ, Mascagni F (1996). Cortico-cortical and corticoamygdaloid projections of the rat occipital cortex: a Phaseolus vulgaris leucoagglutinin study. Neuroscience 71: 37-54.

McLean PD (1955). The limbis system ('visceral brain') and emotional behaviour. Arch Neurol Psychiatry 73: 130-134.

Meunier M, Bachevalier J, Murray EA, Malkova L, Mishkin M (1999). Effects of aspiration versus neurotoxic lesions of the amygdala on emotional responses in monkeys. Eur J Neurosci 11: 4403-4418.

Mitelman SA, Buchsbaum MS, Brickman AM, Shihabuddin L (2005). Cortical intercorrelations of frontal area volumes in schizophrenia. Neuroimage 27: 753-770.

Morgan MA, LeDoux JE (1995). Differential contribution of dorsal and ventral medial prefrontal cortex to the acquisition and extinction of conditioned fear in rats. Behav Neurosci 109: 681-688.

New AS, Hazlett EA, Buchsbaum MS, Goodman M, Reynolds D, Mitropoulou V et al (2002). Blunted prefrontal cortical 18fluorodeoxyglucose positron emission tomography response to meta-chloropiperazine in impulsive aggression. Arch Gen Psychiatry 59: 621-629.

New AS, Trestman RF, Mitropoulou V, Goodman M, Koenigsberg $\mathrm{HH}$, Silverman J et al (2004). Low prolactin response to fenfluramine in impulsive aggression. J Psychiatr Res 38: 223-230.

O'Keane V, Maloney E, O’Neil H, O'Connor A, Smith C, Dinan TG (1992). Blunted prolactin response to d-fenfluramine in sociopathy: evidence for subsensitivity of central serotonergic function. $B r$ J Psychiatry 160: 643-646.

Oldfield RC (1971). The assessment and analysis of handedness: the Edinburgh inventory. Neuropsychologia 9: 97-113.

Pezawas L, Meyer-Lindenberg A, Drabant EM, Verchinski BA, Munoz KE, Kolachana BS et al (2005). 5-HTTLPR polymorphism impacts human cingulate-amygdala interactions: a genetic susceptibility mechanism for depression. Nat Neurosci 8: 828-834.

Pfohl B, Blum N, Zimmerman M (1997). Structured Clinical Interview for DSM-IV Personality (SIDP-IV). American Psychiatric Press: Washington, DC.

Protopopescu X, Pan H, Tuescher O, Cloitre M, Goldstein M, Engelien W et al (2005). Differential time courses and specificity of amygdala activity in posttraumatic stress disorder subjects and normal control subjects. Biol Psychiatry 57: 464-473.

Rosenkranz JA, Grace AA (1999). Modulation of basolateral amygdala neuronal firing and afferent drive by dopamine receptor activation in vivo. J Neurosci 19: 11027-11039.

Rosenkranz JA, Grace AA (2002). Cellular mechanisms of infralimbic and prelimbic prefrontal cortical inhibition and dopaminergic modulation of basolateral amygdala neurons in vivo. J Neurosci 22: 324-337.

Rusch N, van Elst LT, Ludaescher P, Wilke M, Huppertz HJ, Thiel $T$ et al (2003). A voxel-based morphometric MRI study in female patients with borderline personality disorder. Neuroimage 20: 385-392.

Sachdev P, Smith JS, Matheson J, Last P, Blumbergs P (1992). Amygdalo-hippocampectomy for pathological aggression. Aust NZ J Psychiatry 26: 671-676.
Saddoris MP, Gallagher M, Schoenbaum G (2005). Rapid associative encoding in basolateral amygdala depends on connections with orbitofrontal cortex. Neuron 46: 321-331.

Schmahl CG, Elzinga BM, Vermetten E, Sanislow C, McGlashan TH, Bremner JD (2003). Neural correlates of memories of abandonment in women with and without borderline personality disorder. Biol Psychiatry 54: 142-151.

Schoenbaum G, Setlow B, Nugent SL, Saddoris MP, Gallagher M (2003). Lesions of orbitofrontal cortex and basolateral amygdala complex disrupt acquisition of odor-guided discriminations and reversals. Learn Mem 10: 129-140.

Shin LM, Orr SP, Carson MA, Rauch SL, Macklin ML, Lasko NB et al (2004). Regional cerebral blood flow in the amygdala and medial prefrontal cortex during traumatic imagery in male and female Vietnam veterans with PTSD. Arch Gen Psychiatry 61: 168-176.

Shin LM, Wright CI, Cannistraro PA, Wedig MM, McMullin K, Martis B et al (2005). A functional magnetic resonance imaging study of amygdala and medial prefrontal cortex responses to overtly presented fearful faces in posttraumatic stress disorder. Arch Gen Psychiatry 62: 273-281.

Siever LJ, Buchsbaum M, New A, Spiegel-Cohen J, Wei T, Hazlett E et al (1999a). d,l-fenfluramine response in impulsive personality disorder assessed with 18F-deoxyglucose positron emission tomography. Neuropsychopharmacology 20: 413-423.

Siever LJ, Buchsbaum MS, New AS, Spiegel-Cohen J, Wei T, Hazlett EA et al (1999b). d,l-fenfluramine response in impulsive personality disorder assessed with [18F]fluorodeoxyglucose positron emission tomography. Neuropsychopharmacology 20: 413-423.

Sokoloff L (1982). The radioactive deoxyglucose method theory, procedures, and applications for measurement of glucose utilization in the central nervous system. Adv Neurochemistry 4: $1-82$.

Soloff PH, Kelly TM, Strotmeyer SJ, Malone KM, Mann JJ (2003). Impulsivity, gender, and response to fenfluramine challenge in borderline personality disorder. Psychiatry Res 119: 11-24.

Soloff PH, Meltzer CC, Greer PJ, Constantine D, Kelly TM (2000). A fenfluramine-activated FDG-PET study of borderline personality disorder. Biol Psychiatry 47: 540-547.

Somerville LH, Kim H, Johnstone T, Alexander AL, Whalen PJ (2004). Human amygdala responses during presentation of happy and neutral faces: correlations with state anxiety. Biol Psychiatry 55: 897-903.

Stefanacci L, Amaral DG (2002). Some observations on cortical inputs to the macaque monkey amygdala: an anterograde tracing study. J Comp Neurol 451: 301-323.

Swartz MS, Blazer DG, George LK, Winfield I (1990). Estimating the prevalence of borderline personality disorder in the community. J Pers Disor 4: 257-272.

Szabo CA, Lancaster JL, Xiong J, Cook C, Fox P (2003). MR imaging volumetry of subcortical structures and cerebellar hemispheres in normal persons. AJNR Am J Neuroradiol 24: 644-647.

Tebartz van Elst L, Hesslinger B, Thiel T, Geiger E, Haegele K, Lemieux L et al (2003). Frontolimbic brain abnormalities in patients with borderline personality disorder: a volumetric magnetic resonance imaging study. Biol Psychiatry 54: 163-171.

Virkkunen M, Rawlings R, Tokola R, Poland RE, Guidotti A, Nemeroff C et al (1994). CSF biochemistries, glucose metabolism, and diurnal activity rhythms in alcoholic violent offenders, fire setters, and healthy volunteers. Arch Gen Psychiatry 51: 20-27.

Wang Z, Hulihan TJ, Insel TR (1997). Sexual and social experience is associated with different patterns of behavior and neural activation in male prairie voles. Brain Res 767: 321-332.

Whalen PJ, Rauch SL, Etcoff NL, McInerney SC, Lee MB, Jenike MA (1998). Masked presentations of emotional facial expres- 
sions modulate amygdala activity without explicit knowledge. J Neurosci 18: 411-418.

Whalen PJ, Shin LM, McInerney SC, Fischer H, Wright CI, Rauch SL (2001). A functional MRI study of human amygdala responses to facial expressions of fear versus anger. Emotion 1: 70-83.

Wignall EL, Dickson JM, Vaughan P, Farrow TF, Wilkinson ID, Hunter MD et al (2004). Smaller hippocampal volume in patients with recent-onset posttraumatic stress disorder. Biol Psychiatry 56: $832-836$.
Woods R, Mazziota J, Cherry S (1993). MRI-PET registration with automated algorithm. J Comput Assist Tomogr 17: 536-546.

Zbrozyna AW, Westwood DM (1991). Stimulation in prefrontal cortex inhibits conditioned increase in blood pressure and avoidance bar pressing in rats. Physiol Behav 49: 705-708.

Zetzsche T, Frodl T, Preuss UW, Schmitt G, Seifert D, Leinsinger G et al (2006). Amygdala volume and depressive symptoms in patients with borderline personality disorder. Biol Psychiatry 60: 302-310. 